Marketplace is provided as a service to readers using text and images from the manufacturer, supplier or distributor and does not imply endorsement by Vital. Normal and prudent research should be exercised before purchase or use of any product mentioned.

\section{FREE TRIAL}

Bambach is so convinced of the health benefits of its saddle seat it is offering you a no obligation 30-day trial in the comfort of your own surgery.

Using a Bambach seat greatly reduces back strain allowing your spine to function in its optimal, balanced axis, resulting in improved

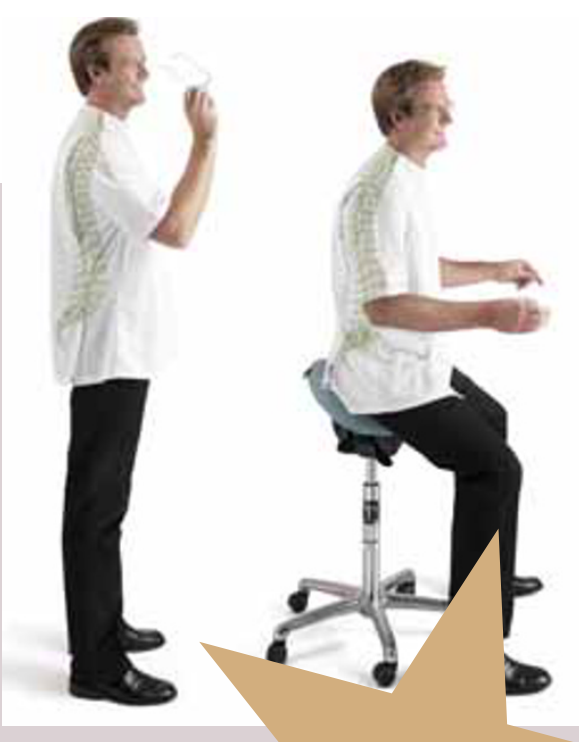

posture and reduced fatigue. This is achieved by maintaining the natural curves of your spine. Every Bambach saddle seat is fully adjustable to create a bespoke stool for individual use.

Endorsed by the Australian Physiotherapy Association, the Bambach Seat is available in a range of ten vinyl colours, 16 hard wearing leathers as well as five pure new wools to perfectly match your décor.

Contact Bambach to arrange a 30-day free trial of the seat in the colour and fabric of your choice on 0800581108 .

\section{SURGERY DESIGN}

Henry Schein's renowned in-house surgery design and planning service ensures you receive tailor-made solutions to make your practice stand out from the crowd.

Thanks to the latest CAD technology Henry Schein can design your practice to your exact specifications, producing a scaled layout and detailing the technical installation and positioning requirements of all your surgery units and equipment.

Henry Schein has created a unique reference guide which enables you to compare products based on price, features and value.

Working in partnership with Henry Schein you can equip your practice with the latest, most advanced equipment at a cost that won't break the bank.

www.henryschein.co.uk

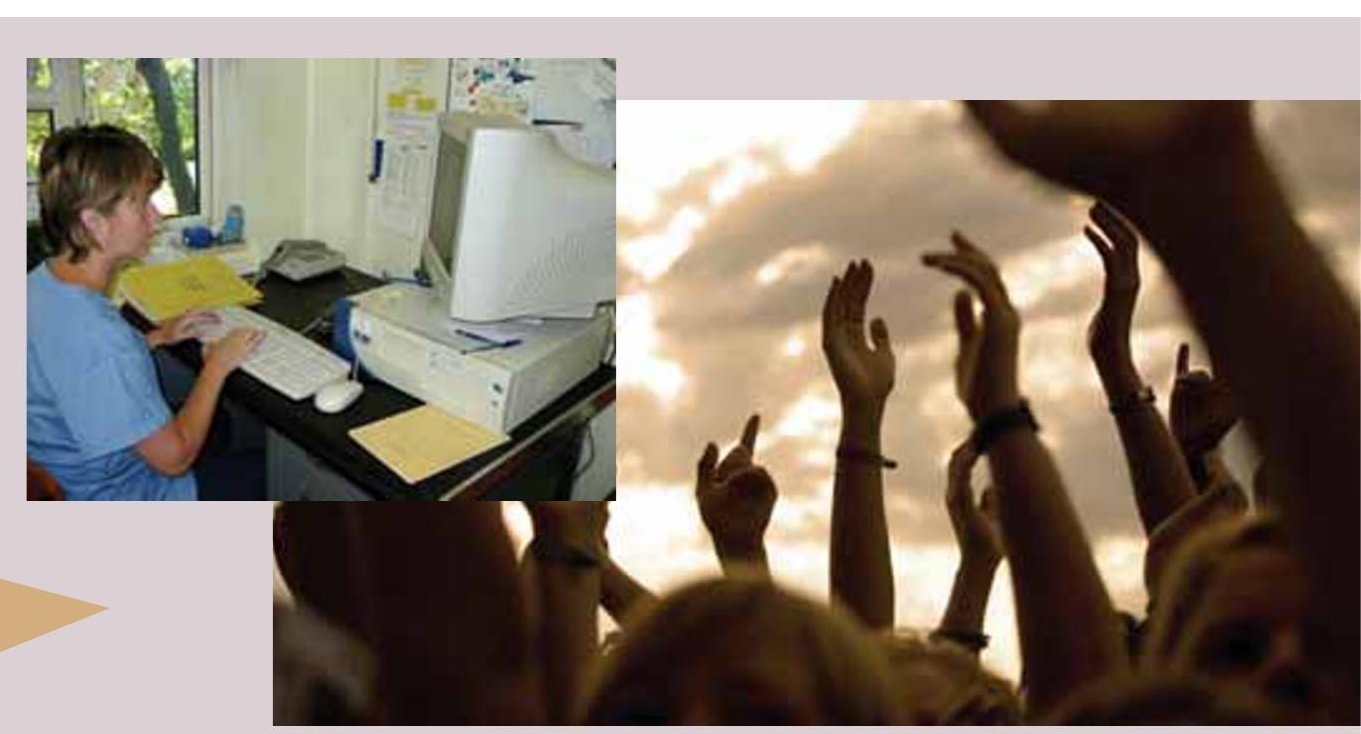

\section{RECRUIT A STAR}

Replacing a key member of staff is not easy. It requires a particular expertise, one that dentists do not always have the time to develop.

Browns Locumlink can locate permanent and locum staff for your practice, and can also provide emergency cover should you lose a team member suddenly. As the specialists at Browns Locumlink carry out extensive interviews with candidates, appraising their ability, education, qualifications and experience, and carry out comprehensive quality-control procedures whenever a candidate completes a placement, you can rest assured that the professionals Browns select will fit right in to your practice.

Browns Locumlink understands the crucial importance of matching candidate and role. Contact Browns Locumlink on 0208927 0972, and let the experts recruit your latest star.

\section{INTRODUCTORY TRAINING}

Smile-On has designed a program to fulfil the GDC's requirement for new dental nurses to be on an appropriate course prior to formal training and later qualification.

The course may also serve as a refresher course for experienced dental nurses who are returning to the profession after a break.

The program ensures that the user will be able to undertake basic surgery duties safely and competently and prepares the user for a career in dental nursing. Users will receive enrolment and completion certificates.

Based on state of the art technology the program has been designed with ease of use in mind and costs $\mathfrak{E} 69$. Dental nurses will gain important introductory training to enable them to progress towards their qualification. Telephone 02074008989 for more information.

\section{RAISE YOUR PROFILE}

Does your practice literature reflect the quality of dental care you provide to your patients? Designer Dental can help transform your literature to boost interest in your services and what is happening at your practice.

Designer Dental is a dental marketing company working exclusively within the dental profession to provide marketing solutions to help elevate the uptake of treatments and encourage patient loyalty. Professional and well designed welcome packs, treatment leaflets, newsletters, posters, referral cards and stationery will help lift the profile of the practice to your patients.

Whether you want to expand and increase your patient base or if you just want to provide the very best information and literature to your patients, contact Designer Dental on 01642206106. 